\title{
Hydrogen escape, Earth's irreversible oxidation, and the Great Oxidation Event revisited
}

\author{
DAVID C. CATLING ${ }^{1}$, SHINTARO KADOYA ${ }^{1}$ AND KEVIN

\section{J. ZANHLE ${ }^{2}$}

${ }^{1}$ University of Washington

${ }^{2}$ NASA Ames Research Center

Presenting Author: dcatling@uw.edu

Two decades ago, we proposed that Earth's early anoxic atmosphere caused rapid escape of hydrogen to space, irreversible oxidation of the Earth, and the Great Oxidation Event (GOE) tipping point [1]. Hydrogen escape is widely accepted as the ultimate cause of Venus' and Mars' oxidized atmospheres and the red color of Mars. But its role as the explanation for Earth's oxic atmosphere and red continental surface (along with photosynthetic $\mathrm{O}_{2}$ ), is still not the standard view. Here, we revisit the theory in light of recent data.

For estimated levels of Archean (biogenic) atmospheric methane, time-integrated hydrogen escape predicts prodigious oxidation beyond that in the crust. Estimates [2] show that oxidized species exceed reduced carbon in the continental crust by $\sim 2$, which cannot result from photosynthesis alone. Also, zircon cerium anomalies indicate a more reducing early crust [3]. New data also show that the Archean upper mantle became progressively oxidized; thus, reducing early volcanic gases would suppress $\mathrm{O}_{2}$ and delay the GOE [4].

Ref. [1] noted that evidence of $\mathrm{H}$ escape might reside in low $\mathrm{D} / \mathrm{H}$ in Archean seawater. Data now suggest that oceanic $\mathrm{D} / \mathrm{H}$ was lower than present [5].

Fractionation of xenon isotopes provides the strongest evidence for $\mathrm{H}$ escape. The isotope distribution becomes heavier through time until around the GOE, plausibly because xenon was dragged by hydrogen escape [6]. Archean Xe isotopes also require intermittent Hadean hydrogen escape, perhaps from impact-induced reducing atmospheres.

Applied to Earth-like exoplanets, $\mathrm{H}$ escape will drive oxidation. Methanogenesis puts reducing power into the air, which otherwise might be sequestered into solids. If oxygenic photosynthesis evolves, $\mathrm{O}_{2}$ can dominate atmospheric redox, producing a remotely observable biosignature and allowing complex life.

[1] Catling, D. C. et al. (2001), Science 293, 839-843.

[2] Hayes, J. M.; Waldbauer (2006), J. R. Phil Trans R Soc B 361, 931-950.

[3] Yang, X. Z. et al. (2014), Earth Planet Sc Lett 393, 210 219.

[4] Kadoya, S. et al. (2020) Nat Commun 11, 2774.

[5] Pope, E. C. et al. (2012) P Natl Acad Sci USA 109, 43714376.

[6] Zahnle, K. J. et al. (2019) Geochim Cosmochim Acta 244, 56-85. 